\title{
ON THE POINTWISE MULTIPLICATION IN BESOV AND LIZORKIN-TRIEBEL SPACES
}

\author{
DOUADI DRIHEM AND MADANI MOUSSAI
}

Received 1 February 2005; Revised 22 February 2006; Accepted 4 April 2006

Under some sufficient conditions satisfied by $F$-space of Lizorkin and Triebel and $B$-space of Besov, we prove some embeddings of types $F \cdot B \hookrightarrow F, F \cdot F \hookrightarrow F$, and $B \cdot B \hookrightarrow B$.

Copyright (c) 2006 Hindawi Publishing Corporation. All rights reserved.

\section{Introduction and preparations}

In Besov spaces and Lizorkin-Triebel spaces, this paper is concerned with proving some embeddings of the form

$$
F \cdot B \hookrightarrow F, \quad F \cdot F \hookrightarrow F, \quad B \cdot B \hookrightarrow B,
$$

where $F$ and $B$, with three indices, will denote the Lizorkin-Triebel space $F_{p, q}^{s}$ and the Besov space $B_{p, q}^{s}$, respectively. The different embeddings obtained here are under certain restrictions on the parameters.

In this introduction, we will recall the definition of some spaces and some necessary tools. In Sections 2 and 3, we give the first contribution of this work. The theorems of Section 2 will treat the case $F \cdot B \hookrightarrow F$ where the first theorem is a generalization of the results of Franke [4, Section 3.2, Theorem 1, Section 3.4, Corollary 1] and Marschall [7]. The second theorem is in the sense of Johnsen's works (see [5]). Section 3 will contain a treatment of the embeddings of the types $F \cdot F \hookrightarrow F$ and $B \cdot B \hookrightarrow B$ which presents an improvement of [3].

In the sense of [5, Theorems 6.5, 6.11], some limit cases are considered in Section 4, which constitute the second contribution of this paper. Section 5 is an application of our results to the continuity of pseudodifferential operators on Lizorkin-Triebel spaces.

We will work on the Euclidean space $\mathbb{R}^{n}$. If $f \in \mathscr{Y}$, the Fourier transform is defined by the formula

$$
\mathscr{F} f(\xi)=\hat{f}(\xi)=\int_{\mathbb{R}^{n}} f(x) e^{-i x \cdot \xi} d x \quad\left(\xi \in \mathbb{R}^{n}\right)
$$

Hindawi Publishing Corporation International Journal of Mathematics and Mathematical Sciences Volume 2006, Article ID 76182, Pages 1-18

DOI 10.1155/IJMMS/2006/76182 
2 Multiplication in Besov and Lizorkin spaces

and $\mathscr{F}^{-1} f$ denotes the inverse Fourier transform of $f$; as usual $\mathscr{F}$ and $\mathscr{F}^{-1}$ are extended from $\mathscr{S}$ to $\mathscr{S}^{\prime}$.

Consider a partition of unity

$$
\psi(\xi)+\sum_{j=1}^{\infty} \varphi\left(2^{-j} \xi\right)=1 \quad\left(\xi \in \mathbb{R}^{n}\right)
$$

where $\varphi, \psi \in C^{\infty}$ are positive functions such that $\operatorname{supp} \varphi \subset\left\{\xi \in \mathbb{R}^{n}: 1 \leq|\xi| \leq 3\right\}$ and $\operatorname{supp} \psi \subset\left\{\xi \in \mathbb{R}^{n}:|\xi| \leq 3\right\}$. We define the convolution operators $Q_{j}$ and $\Delta_{k}$ by the following:

$$
\begin{aligned}
& Q_{j} f=\mathscr{F}^{-1}\left(\psi\left(2^{-j} \cdot\right)\right) * f \quad(j=1,2, \ldots), \\
& \Delta_{k} f=\mathscr{F}^{-1}\left(\varphi\left(2^{-k} \cdot\right)\right) * f \quad(k=0,1, \ldots),
\end{aligned}
$$

and we set $Q_{0}=\Delta_{0}$. Thus we obtain the Littlewood-Paley decomposition $f=\sum_{j=0}^{\infty} \Delta_{j} f$ (convergence in $\mathscr{S}^{\prime}$ ).

Let us now recall the definitions of $F_{p, q}^{s}$ and $B_{p, q}^{s}$, where the general references include $[1,9-13]$.

Definition 1.1. Let $\gamma>0,-\infty<s<\infty, 0<p<\infty$ (resp., $0<p \leq \infty$ ), and $0<q \leq \infty$. The space $L_{p}^{\gamma}\left(\ell_{q}^{s}\right)$ (resp., $\left.\ell_{q}^{s}\left(L_{p}^{\gamma}\right)\right)$ is the set of the sequences $\left\{f_{k}\right\}_{k \in \mathbb{N}} \subset S^{\prime}$ such that supp $\hat{f}_{k} \subset$ $\left\{\xi \in \mathbb{R}^{n}:|\xi|<\gamma 2^{k}\right\}$ and

$$
\begin{gathered}
\left\|\left\{f_{k}\right\}_{k \in \mathbb{N}}\left|L_{p}^{\gamma}\left(\ell_{q}^{s}\right)\|=\|\left\{2^{k s} f_{k}\right\}_{k \in \mathbb{N}}\right| L_{p}\left(\ell_{q}\right)\right\|<\infty, \\
\text { (resp., } \left.\left\|\left\{f_{k}\right\}_{k \in \mathbb{N}}\left|\ell_{q}^{s}\left(L_{p}^{\gamma}\right)\|=\|\left\{2^{k s} f_{k}\right\}_{k \in \mathbb{N}}\right| \ell_{q}\left(L_{p}\right)\right\|<\infty\right) .
\end{gathered}
$$

Definition 1.2. (i) Let $0<p<\infty, 0<q \leq \infty$, and $-\infty<s<\infty$, then

$$
F_{p, q}^{s}=\left\{f \in \mathscr{S}^{\prime}:\left\|\left\{2^{k s} \Delta_{k} f\right\}_{k \in \mathbb{N}} \mid L_{p}\left(\ell_{q}\right)\right\|<\infty\right\} .
$$

(ii) Let $0<p, q \leq \infty$, and $-\infty<s<\infty$, then

$$
B_{p, q}^{s}=\left\{f \in \mathscr{S}^{\prime}:\left\|\left\{2^{k s} \Delta_{k} f\right\}_{k \in \mathbb{N}} \mid \ell_{q}\left(L_{p}\right)\right\|<\infty\right\} .
$$

Remark 1.3. We introduce the maximal function

$$
\Delta_{k}^{*, a} f(x)=\sup _{y \in \mathbb{R}^{n}} \frac{\left|\Delta_{k} f(x-y)\right|}{1+\left(2^{k}|y|\right)^{a}}
$$

for all $x \in \mathbb{R}^{n}, f \in \mathscr{S}^{\prime}, a>0$, and $k=0,1, \ldots$ Then, in Definition 1.2(i) (resp., (ii)), we can replace $\Delta_{k} f$ by $\Delta_{k}^{*, a} f$ with $a>(n / \min (p, q))$ (resp., $\left.a>n / p\right)$, (cf. see [13, Theorem 2.3.2]). 
The product $f \cdot g$ is defined by

$$
f \cdot g=\lim _{j \rightarrow \infty} Q_{j} f \cdot Q_{j} g \quad\left(\forall f, g \in \mathscr{S}^{\prime}\right)
$$

if the limit on the right-hand side exists in $\mathscr{S}^{\prime}$ (see [10, Section 4.2]), and we have

$$
\Delta_{k}(f \cdot g)=\sum_{j, \ell=0}^{\infty} \Delta_{k}\left(\Delta_{j} g \cdot \Delta_{\ell} f\right)=\left(\Pi_{k, 1}+\Pi_{k, 2}+\Pi_{k, 3}\right)(f, g),
$$

where

$$
\begin{gathered}
\Pi_{k, 1}(f, g)=\Delta_{k}\left(\tilde{\Delta}_{k} f \cdot Q_{k+1} g\right), \quad \Pi_{k, 2}(f, g)=\Delta_{k}\left(Q_{k+1} f \cdot \tilde{\Delta}_{k} g\right), \\
\Pi_{k, 3}(f, g)=\sum_{j=k}^{\infty} \Delta_{k}\left(\Delta_{j} f \cdot \bar{\Delta}_{j} g\right),
\end{gathered}
$$

with $\widetilde{\Delta}_{k}=\sum_{j=k-2}^{k+4} \Delta_{j}$ and $\bar{\Delta}_{k}=\sum_{j=k-1}^{k+1} \Delta_{j}$.

In the below proofs of the different cases of type (1.1), written as $G_{1} \cdot G_{2} \hookrightarrow G_{3}$, to see $f \cdot g$ belongs to $G_{3},\left(f \in G_{1}, g \in G_{2}\right)$, it suffices to an estimate of terms of the form $\left\|\left\{\Pi_{k, i}(f, g)\right\}_{k \in \mathbb{N}} \mid L_{p}^{\gamma}\left(\ell_{q}^{s}\right)\right\|$ and $\left\|\left\{\Pi_{k, i}(f, g)\right\}_{k \in \mathbb{N}} \mid \ell_{q}^{s}\left(L_{p}^{\gamma}\right)\right\|, i \in\{1,2,3\}$.

Now we recall some lemmas which are useful for us.

Lemma 1.4. (i) Let $-\infty<s_{i}<\infty, 0<p_{i}<\infty$ (resp., $0<p_{i} \leq \infty$ ), and $0<q_{i} \leq \infty$ (with $i=0,1)$. If

$$
s_{0}>s_{1}, \quad p_{0}=p_{1},
$$

or

$$
s_{0} \geq s_{1}, \quad s_{0}-\frac{n}{p_{0}}=s_{1}-\frac{n}{p_{1}} \quad\left(q_{0} \leq q_{1} \text { for Besov space }\right)
$$

then it holds

$$
\left.F_{p_{0}, q_{0}}^{s_{0}} \hookrightarrow F_{p_{1}, q_{1}}^{s_{1}} \quad \text { (resp., } B_{p_{0}, q_{0}}^{s_{0}} \hookrightarrow B_{p_{1}, q_{1}}^{s_{1}}\right) .
$$

(ii) Let $-\infty<s, s_{i}<\infty, 0<p, p_{i}<\infty$, and $0<q, q_{i} \leq \infty$ (with $\left.i=0,1\right)$ such that $s_{0}-$ $n / p_{0}=s-n / p=s_{1}-n / p_{1}$. If

$$
s_{0}>s>s_{1}, \quad q_{0} \leq p \leq q_{1},
$$

or

$$
s_{0}=s=s_{1}, \quad q_{0} \leq \min (p, q), \quad q_{1} \geq \max (p, q),
$$

then it holds

$$
B_{p_{0}, q_{0}}^{s_{0}} \hookrightarrow F_{p, q}^{s} \hookrightarrow B_{p_{1}, q_{1}}^{s_{1}} .
$$


4 Multiplication in Besov and Lizorkin spaces

(iii) Let $-\infty<s<\infty, 0<p<\infty$ (resp., $0<p \leq \infty$ ), and $0<q \leq \infty$. If

$$
s>\frac{n}{p}
$$

or

$$
s=\frac{n}{p}, \quad 0<p \leq 1(\text { resp., } 0<q \leq 1)
$$

then it holds

$$
F_{p, q}^{s} \hookrightarrow L_{\infty} \quad\left(\text { resp., } B_{p, q}^{s} \hookrightarrow L_{\infty}\right) \text {. }
$$

Lemma 1.5. Let $0<\gamma<1$ and $0<q \leq \infty$. Let $\left\{\varepsilon_{k}\right\}_{k \in \mathbb{N}}$ be a sequence of positive real numbers such that $\left\|\left\{\varepsilon_{k}\right\}_{k \in \mathbb{N}} \mid \ell_{q}\right\|=A<\infty$. Then the sequences $\delta_{k}=\sum_{j=0}^{k} \gamma^{k-j} \varepsilon_{j}$ and $\eta_{k}=$ $\sum_{j=k}^{\infty} \gamma^{j-k} \varepsilon_{j}$ belong to $\ell_{q}$, and the estimate

$$
\left\|\left\{\delta_{k}\right\}_{k \in \mathbb{N}}\left|\ell_{q}\|+\|\left\{\eta_{k}\right\}_{k \in \mathbb{N}}\right| \ell_{q}\right\| \leq c A
$$

holds. The constant $c$ depends only on $\gamma$ and $q$.

Lemma 1.6. Let $0<p \leq \infty$ and $\gamma>0$. Let $\left\{f_{j}\right\}_{j \in \mathbb{N}} \subset L_{p}$ be a sequence of functions such that $\operatorname{supp} \hat{f}_{j} \subset\left\{\xi \in \mathbb{R}^{n}:|\xi| \leq \gamma 2^{j}\right\}$. Then the estimate

$$
\left\|\Delta_{k} f_{j}\left|L_{p}\left\|\leq c 2^{(j-k) \varrho}\right\| f_{j}\right| L_{p}\right\| \quad\left(k \leq j<\infty, \varrho=\max \left(0, \frac{n}{p}-n\right)\right)
$$

holds. The constant $c$ depends only on $n, p$, and $\gamma$.

Lemma 1.7. Let $0<p<1$ and $\gamma>0$. Let $\left\{f_{j}\right\}_{j \in \mathbb{N}} \subset L_{p}$ be a sequence of functions such that $\operatorname{supp} \hat{f}_{j} \subset\left\{\xi \in \mathbb{R}^{n}:|\xi| \leq \gamma 2^{j}\right\}$. Then the estimate

$$
\left\|\sum_{j=0}^{\infty} f_{j}\left|B_{p, \infty}^{\varrho}\|\leq c\|\left\{2^{j \varrho} f_{j}\right\}_{j \in \mathbb{N}}\right| L_{p}\left(\ell_{\infty}\right)\right\| \quad\left(\varrho=\frac{n}{p}-n\right)
$$

holds. The constant $c$ depends only on $n, p$, and $\gamma$.

Lemma 1.8. Let $0<p \leq q \leq \infty$ and $\gamma>0$. Then there exists a constant $c=c(n, p, q)>0$ such that for all $f \in L_{p}$ with supp $\hat{f} \subset\left\{\xi \in \mathbb{R}^{n}:|\xi| \leq \gamma\right\}$, one has

$$
\left\|f\left|L_{q}\left\|\leq c \gamma^{n(1 / p-1 / q)}\right\| f\right| L_{p}\right\| \text {. }
$$

For Lemma 1.4, we can see [11, Sections 2.3 and 2.8] and [12, Section 2.7]. Lemma 1.5 follows from Young's inequality in $\ell_{q}$. The proof of Lemma 1.6 is given in [4, Section 2.4, Theorem 1(iii)] and Lemma 1.7 in [7, Lemma 3]. For the proof of Lemma 1.8, we can see [14, Proposition 2.13], $1 \leq p \leq q \leq \infty$, it is the classical inequality of Bernstein. 


\section{Multiplication of mixed type}

The following results give an extension of the sufficient hypotheses used in [5, Theorem 6.1].

Theorem 2.1. Let $0<p, p_{1}, p_{2}<\infty, 0<q, q_{2} \leq \infty,-\infty<s<\infty$, and $r>0$ be such that

$$
\begin{gathered}
-r+\max \left(0, \frac{n}{p_{1}}+\frac{n}{p_{2}}-n\right)<s<\min \left(\frac{n}{p_{1}}, r\right), \\
\frac{1}{p}=\frac{1}{p_{1}}+\frac{1}{p_{2}}-\frac{r}{n}, \quad \frac{1}{q_{2}} \geq \frac{1}{p_{2}}-\frac{r}{n}, \quad r<\frac{n}{p_{2}}\left(\text { resp., } r=\frac{n}{p_{2}}\right) .
\end{gathered}
$$

Then it holds

$$
F_{p_{1}, q}^{s} \cdot B_{p_{2}, q_{2}}^{r} \hookrightarrow F_{p, q}^{s} \quad\left(\text { resp., } F_{p_{1}, q}^{s} \cdot\left(B_{p_{2}, \infty}^{n / p_{2}} \cap L_{\infty}\right) \hookrightarrow F_{p_{1}, q}^{s}\right) .
$$

Corollary 2.2. Under the hypotheses of Theorem 2.1. If $r<n / p_{2}\left(\right.$ resp., $\left.r=n / p_{2}\right)$ then it holds

$$
F_{p_{1}, q}^{s} \cdot F_{p_{2}, q_{2}}^{r} \hookrightarrow F_{p, q}^{s} \quad\left(\text { resp., } F_{p_{1}, q}^{s} \cdot F_{p_{2}, q_{2}}^{n / p_{2}} \hookrightarrow F_{p_{1}, q}^{s} \text { for } p_{2} \leq 1\right) .
$$

Furthermore, in particular, if $1<p_{1}<\infty$ and $r>n / p_{1}+n / p_{2}-n$, can be taken $s=0$ in (2.3).

Proof. Since $F_{p_{2}, q_{2}}^{r} \hookrightarrow B_{p_{2}, t}^{r}$ with $t=\left(1 / p_{2}-r / n\right)^{-1}$, we obtain the first embedding. However, the second embedding follows from $F_{p_{2}, q_{2}}^{n / p_{2}} \hookrightarrow B_{p_{2}, \infty}^{n / p_{2}} \cap L_{\infty}$.

Remark 2.3. In Corollary 2.2, when $r<n / p_{2}$ (resp., $r=n / p_{2}$ ), we obtain [10, Theorems 4.4.3/2(21) and 4.4.4/2(16) (resp., Theorems 4.4.3/2(22) and 4.4.4/2(17))]. The particular case $s=0$ presents a complement of [10, Theorem 4.4.4/4(i)].

To prove Theorem 2.1, we need the following lemma.

Lemma 2.4. Let $0<p<\infty$ and $a>n / p$. Then there exists a constant $c>0$ such that

$$
\left\|\left\{Q_{j}^{*, a} g\right\}_{j \in \mathbb{N}}\left|L_{p}\left(\ell_{\infty}\right)\|\leq c\| g\right| F_{p, 2}^{0}\right\|,
$$

for any $g \in F_{p, 2}^{0}$.

Proof. First, we define the maximal function of $Q_{j} g$, of Hardy-Littelewood type, by the formula

$$
M Q_{j} g(x)=\sup _{r>0} \frac{1}{|B(x, r)|} \int_{B(x, r)}\left|Q_{j} g(y)\right| d y,
$$

where $B(x, r)$ is the ball centered at $x$ of radius $r$ and $|B(x, r)|$ denotes its measure. Next, let $t>0$ satisfy $n / a<t<p$. From [13, Theorem 1.3.1], we have

$$
Q_{j}^{*, a} g(x) \leq Q_{j}^{*, n / t} g(x) \leq c\left(M\left|Q_{j} g\right|^{t}(x)\right)^{1 / t} \quad\left(\forall x \in \mathbb{R}^{n}\right) .
$$


6 Multiplication in Besov and Lizorkin spaces

Then we obtain

$$
\begin{aligned}
\left\|\sup _{j \in \mathbb{N}} Q_{j}^{*, a} g \mid L_{p}\right\| & \leq c\left\|\left.\left(\sup _{j \in \mathbb{N}} M\left|Q_{j} g\right|^{t}\right)^{1 / t}\left|L_{p}\|=c\| \sup _{j \in \mathbb{N}} M\right| Q_{j} g\right|^{t} \mid L_{p / t}\right\|^{1 / t} \\
& \leq c^{\prime}\left\|\sup _{j \in \mathbb{N}}\left|Q_{j} g\right|^{t} \mid L_{p / t}\right\|^{1 / t} .
\end{aligned}
$$

A proof of the last inequality may be found in [13, Theorem 2.2.2, page 89]. Now, it is easy to see that the last member of (2.7) is bounded by

$$
\left\|\sup _{j \in \mathbb{N}}\left|Q_{j} g\right|\left|L_{p}\|\leq c\| g\right| F_{p, 2}^{0}\right\| .
$$

Inequality (2.8) follows from the equality between the local Hardy spaces $h_{p}$ and $F_{p, 2}^{0}$, (cf. see [12, Section 2.2, page 37, and Theorem 2.5.8/1]).

Proof of Theorem 2.1

Case $1\left(r<n / p_{2}\right)$. (i) Estimate of $\left\{\Pi_{k, 1}(f, g)\right\}_{k \in \mathbb{N}}$. Since

$$
\begin{aligned}
\left|\Pi_{k, 1}(f, g)(x)\right| & =\left|\int_{\mathbb{R}^{n}}\left(\mathscr{F}^{-1} \varphi(y)\right)\left(Q_{k+1} g \cdot \tilde{\Delta}_{k} f\right)\left(x-2^{-k} y\right) d y\right| \\
& \leq c Q_{k+1}^{*, a_{1}} g(x) \tilde{\Delta}_{k}^{*, a_{2}} f(x) \quad\left(\forall x \in \mathbb{R}^{n}\right),
\end{aligned}
$$

where $Q_{k}^{*, a_{1}}$ and $\widetilde{\Delta}_{k}^{*, a_{2}}$ are defined as in Remark 1.3, we obtain

$$
\left\|\left\{2^{k s} \Pi_{k, 1}(f, g)\right\}_{k \in \mathbb{N}}\left|\ell_{q}\left\|\leq \sup _{j \in \mathbb{N}}\left(Q_{j}^{*, a_{1}} g\right)\right\|\left\{2^{k s} \tilde{\Delta}_{k}^{*, a_{2}} f\right\}_{k \in \mathbb{N}}\right| \ell_{q}\right\|,
$$

where $a_{1}$ and $a_{2}$ are real numbers at our disposal. We set $1 / b=1 / p_{2}-r / n$. The left-hand side of (2.10), in $L_{p}$-norm, is bounded by

$$
c\left\|\sup _{j \in \mathbb{N}} Q_{j}^{*, a_{1}} g\left|L_{b}\|\|\left\{2^{k s} \tilde{\Delta}_{k}^{*, a_{2}} f\right\}_{k \in \mathbb{N}}\right| L_{p_{1}}\left(\ell_{q}\right)\right\| .
$$

Choose $a_{1}>n / b$ and $a_{2}>n / \min \left(p_{1}, q\right)$, then both Lemma 2.4 and the embedding $B_{p_{2}, q_{2}}^{r} \hookrightarrow$ $F_{b, 2}^{0}$ yield that (2.11) is estimated as desired.

(ii) Estimate of $\left\{\prod_{k, 2}(f, g)\right\}_{k \in \mathbb{N}}$. Let $u \in \mathbb{R}$ such that

$$
\max \left(0, \frac{1}{p_{1}}-\frac{r}{n}\right)<\frac{1}{u}<\min \left(\frac{1}{p_{1}}, \frac{1}{p_{1}}-\frac{s}{n}\right) .
$$

We set

$$
\frac{1}{v}=\frac{1}{p_{2}}+\frac{1}{u}, \quad \sigma=s-\frac{n}{p}+\frac{n}{v}, \quad \beta=s-\frac{n}{p_{1}}+\frac{n}{u} .
$$


We have

$$
\ell_{p}^{\sigma}\left(L_{v}^{\gamma}\right) \hookrightarrow L_{p}^{\gamma}\left(\ell_{q}^{s}\right), \quad F_{p_{1}, q}^{s} \hookrightarrow B_{u, p_{1}}^{\beta} .
$$

For the first embedding of (2.14), we can see [4, Section 2.3, Theorem 3]. On the other hand, the Hölder inequality yields

$$
2^{k \sigma}\left\|\Pi_{k, 2}(f, g)\left|L_{v}\left\|\leq c 2^{k r}\right\| \widetilde{\Delta}_{k} g\right| L_{p_{2}}||\left(2^{k \beta} \sum_{j=0}^{k+1} 2^{-j \beta} \cdot 2^{j \beta}\left\|\Delta_{j} f \mid L_{u}\right\|\right) .\right.
$$

We set $1 / \tilde{q}_{2}=1 / p-1 / p_{1}$. Applying, successively, the Hölder inequality again in $\ell_{p}$-norm and Lemma 1.5, we obtain the bound $c\left\|g\left|B_{p_{2}, \tilde{q}_{2}}^{r}\|\| f\right| B_{u, p_{1}}^{\beta}\right\|$. So (2.14) and $B_{p_{2}, q_{2}}^{r} \hookrightarrow B_{p_{2}, \tilde{q}_{2}}^{r}$ give

$$
\left\|\left\{2^{k s} \Pi_{k, 2}(f, g)\right\}_{k \in \mathbb{N}}\left|L_{p}\left(\ell_{q}\right)\|\leq c\| g\right| B_{p_{2}, q_{2}}^{r}\right\|\left\|f \mid F_{p_{1}, q}^{s}\right\| .
$$

(iii) Estimate of $\left\{\Pi_{k, 3}(f, g)\right\}_{k \in \mathbb{N}}$. We first consider $1 / p_{1}+1 / p_{2} \leq 1$. Let $u \in \mathbb{R}$ such that

$$
\max \left(0, \frac{1}{p_{1}}-\frac{r}{n}, \frac{1}{p_{1}}-\frac{r+s}{n}\right)<\frac{1}{u}<\frac{1}{p_{1}} .
$$

We use the notations $v, \sigma$, and $\beta$ from (2.13). Lemma 1.6 provides

$$
2^{k \sigma}\left\|\Pi_{k, 3}(f, g)\left|L_{v}\left\|\leq c 2^{k(\beta+r)} \sum_{j=k}^{\infty} 2^{-j(\beta+r)} \cdot 2^{j(\beta+r)}\right\| \bar{\Delta}_{j} g\right| L_{p_{2}}\right\|\left\|\Delta_{j} f \mid L_{u}\right\| .
$$

A similar argument as above yields

$$
\left\|\left\{2^{k \sigma} \Pi_{k, 3}(f, g)\right\}_{k \in \mathbb{N}}\left|\ell_{p}\left(L_{v}\right)\|\leq c\|\left\{2^{j(\beta+r)}\left\|\bar{\Delta}_{j} g\left|L_{p_{2}}\right|\right\|\left\|\Delta_{j} f \mid L_{u}\right\|\right\}_{j \in \mathbb{N}}\right| \ell_{p}\right\| .
$$

We set $1 / \tilde{q}_{2}=1 / p-1 / p_{1}$. By the Hölder inequality in $\ell_{p}$-norm, the right-hand side of (2.19) is bounded by $c\left\|g\left|B_{p_{2}, \tilde{q}_{2}}^{r}\|\| f\right| B_{u, p_{1}}^{\beta}\right\|$. Then we conclude the desired estimate by (2.14).

We now study case $1 / p_{1}+1 / p_{2}>1$. Let $u \in \mathbb{R}$ such that

$$
\max \left(0,1-\frac{1}{p_{2}}, \frac{1}{p_{1}}-\frac{r}{n}\right)<\frac{1}{u}<\frac{1}{p_{1}} .
$$

We employ the notations $v$ and $\sigma$ from (2.13). By Lemma 1.6, we obtain

$$
2^{k \sigma}|| \Pi_{k, 3}(f, g)\left|L_{v}\left\|\leq c 2^{k \mu} \sum_{j=k}^{\infty} 2^{-j \mu} \cdot 2^{j(r+\varrho)}\right\| \bar{\Delta}_{j} g\right| L_{p_{2}}\left|\|\| \Delta_{j} f\right| L_{u} \|,
$$

where $\varrho=s-n / p_{1}+n / u$ and $\mu=s+r-n / p_{1}-n / p_{2}+n>0$, therefore,

$$
\left\|\left\{2^{k \sigma} \Pi_{k, 3}(f, g)\right\}_{k \in \mathbb{N}}\left|\ell_{p}\left(L_{v}\right)\|\leq c\|\left\{2^{j(r+\varrho)}\left\|\bar{\Delta}_{j} g\left|L_{p_{2}}\|\| \Delta_{j} f\right| L_{u}\right\|\right\}_{j \in \mathbb{N}}\right| \ell_{p}\right\| .
$$


On the right-hand side of (2.22), we employ the Hölder inequality in $\ell_{p}$-norm (with $\left.1 / p=1 / p_{1}+1 / \tilde{q}_{2}\right), F_{p_{1}, q}^{s} \hookrightarrow B_{u, p_{1}}^{\varrho}$, and $B_{p_{2}, q_{2}}^{r} \hookrightarrow B_{p_{2}, \tilde{q}_{2}}^{r}$ successively. Since $\sigma>s$ and $v<p$, we can finish the proof of this case by applying, in the left-hand side of (2.22), embeddings (2.14).

Case $2\left(r=n / p_{2}\right)$. We only estimate $\left\{\Pi_{k, 1}(f, g)\right\}_{k \in \mathbb{N}}$. It is sufficient to see that

$$
2^{k s}\left|\Pi_{k, 1}(f, g)\right| \leq c|| g \mid L_{\infty} \|\left(2^{k s} \tilde{\Delta}_{k}^{*, a} f\right)
$$

with $a>n / \min \left(p_{1}, q\right)$ and to take the $L_{p_{1}}\left(\ell_{q}\right)$-norm.

Theorem 2.5. Let $0<p, p_{1}<\infty, 0<p_{2}, q \leq \infty,-\infty<s<\infty$, and $r>0$ be such that

$$
-r+\max \left(0, \frac{n}{p_{1}}+\frac{n}{p_{2}}-n\right)<s<r .
$$

If either of the following assertions is satisfied:

(i) $1 / p=1 / p_{1}+1 / p_{2}$,

(ii) $\max \left(1 / p_{1}, s / n\right)+\max \left(0,1 / p_{2}-r / n\right)<1 / p<1 / p_{1}+1 / p_{2}$,

then it holds

$$
F_{p_{1}, q}^{s} \cdot B_{p_{2}, \infty}^{r} \hookrightarrow F_{p, q}^{s} \text {. }
$$

Corollary 2.6. Let $p, p_{1}, q, r, s$ be as in Theorem 2.5 and $0<p_{2}<\infty$. If (i) or (ii) of Theorem 2.5 is satisfied, then the embedding $F_{p_{1}, q}^{s} \cdot F_{p_{2}, \infty}^{r} \hookrightarrow F_{p, q}^{s}$ holds.

The proof of Corollary 2.6 is immediate because $F_{p_{2}, \infty}^{r} \hookrightarrow B_{p_{2}, \infty}^{r}$.

Remark 2.7. We note that Theorem 2.5(i) when $p_{2}=\infty$ is given in [4, Section 3.2, Theorem 1]. Also, we note that Corollary 2.6 is given in both [5, Theorem 6.1 with $r=p$ in formula (6.6)] and [10, Theorems 4.4.3/1(7) and 4.4.4/1(7)].

Proof of Theorem 2.5(i). Noting Remark 2.7, we only need to treat the part $0<p_{2}<\infty$.

(i) Estimate of $\left\{\Pi_{k, 1}(f, g)\right\}_{k \in \mathbb{N}}$. From (2.9) and Lemma 2.4, we have

$$
\left\|\left\{2^{k s} \Pi_{k, 1}(f, g)\right\}_{k \in \mathbb{N}}\left|L_{p}\left(\ell_{q}\right)\|\leq c\| g\right| F_{p_{2}, 2}^{0}\right\|\left\|f \mid F_{p_{1}, q}^{s}\right\| .
$$

By embeddings $B_{p_{2}, \infty}^{r} \hookrightarrow B_{p_{2}, \min \left(p_{2}, 2\right)}^{0} \hookrightarrow F_{p_{2}, 2}^{0}$, we obtain that the last term of $(2.26)$ is bounded by the desired quantity.

(ii) Estimate of $\left\{\Pi_{k, 2}(f, g)\right\}_{k \in \mathbb{N}}$. The Hölder inequality provides

$$
\left\|\Pi_{k, 2}(f, g)\left|L_{p}\left\|\leq c\left(2^{-k r} \sum_{j=0}^{k+1} 2^{-j(s-r)} \cdot 2^{-j r}\right)\right\| g\right| B_{p_{2}, \infty}^{r}\right\|\left\|f \mid B_{p_{1}, \infty}^{s}\right\| .
$$

The hypothesis $s<r$ yields

$$
\left\|\left\{2^{k s} \Pi_{k, 2}(f, g)\right\}_{k \in \mathbb{N}}\left|\ell_{\min (p, q)}\left(L_{p}\right)\|\leq c\| g\right| B_{p_{2}, \infty}^{r}\right\|\left\|f \mid B_{p_{1}, \infty}^{s}\right\| .
$$


Using embeddings

$$
\ell_{\min (p, q)}^{s}\left(L_{p}^{\gamma}\right) \hookrightarrow L_{p}^{\gamma}\left(\ell_{q}^{s}\right), \quad F_{p_{1}, q}^{s} \hookrightarrow B_{p_{1}, \infty}^{s},
$$

we obtain the desired result.

(iii) Estimate of $\left\{\Pi_{k, 3}(f, g)\right\}_{k \in \mathbb{N}}$. We set $\varrho=s+r-\max (0, n / p-n)$. Using Lemma 1.6, we obtain

$$
\begin{aligned}
2^{k s}|| \Pi_{k, 3}(f, g) \mid L_{p} \| & \leq c 2^{-k r} \cdot 2^{k \varrho} \sum_{j=k}^{\infty} 2^{-j \varrho}\left(2^{j r} \| \bar{\Delta}_{j} g\left|L_{p_{2}}\right| \mid\right)\left(2^{j s}\left\|\Delta_{j} f \mid L_{p_{1}}\right\|\right) \\
& \leq c 2^{-k r}\left\|g\left|B_{p_{2}, \infty}^{r}\|\| f\right| B_{p_{1}, \infty}^{s}\right\| .
\end{aligned}
$$

In this inequality, we take $\ell_{\min (p, q)}$-norm and we conclude the desired estimate using (2.29).

Proof of Theorem 2.5(ii). (1) Estimate of $\left\{\Pi_{k, 1}(f, g)\right\}_{k \in \mathbb{N}}$. We set $1 / u=1 / p-1 / p_{1}$. As in (2.26), we have the bound $c\left\|g\left|F_{u, 2}^{0}\|\| f\right| F_{p_{1}, q}^{s}\right\|$ which, by the embeddings $B_{p_{2}, \infty}^{r} \hookrightarrow$ $B_{p_{2}, u}^{n / p_{2}-n / u} \hookrightarrow F_{u, 2}^{0}$, is estimated as desired.

(2) Estimate of $\left\{\Pi_{k, 2}(f, g)\right\}_{k \in \mathbb{N}}$. In part, for technical reasons, we prove this in three separate cases:

Case $1(s<0)$. By Lemma 1.6, the Hölder inequality, and Lemma 1.8, we have

$$
\left\|\Pi_{k, 2}(f, g)\left|L_{p}\left\|\leq c 2^{\left(n / p_{1}+n / p_{2}-n / p-r-s\right) k}\right\| g\right| B_{p_{2}, \infty}^{r}\right\|\left\|f \mid B_{p_{1}, \infty}^{s}\right\| .
$$

Since $n / p_{1}+n / p_{2}-n / p-r<0$, we obtain an inequality of type (2.28) and finish the proof of this case using (2.29).

Case $2\left(0 \leq s<n / p_{1}\right)$. We set $1 / b=1 / p_{2}+1 / p_{1}-s / n$. We continue with the following subcases.

Subcase $2.1\left(r \leq n / p_{2}\right.$ and $p \leq b\left(\right.$ or $s \leq n / p_{2}<r$ and $\left.\left.p \leq b\right)\right)$. As in Case 1, we have

$$
\left\|\Pi _ { k , 2 } ( f , g ) \left|L_{p}\left\|\leq c \gamma_{k} 2^{-k r}|| g\left|B_{p_{2}, \infty}^{r}\|\| f\right| B_{p_{1}, \infty}^{s}\right\|\right.\right.
$$

where

$$
\gamma_{k}= \begin{cases}k+2 & \text { if } p=b \\ \left(1-2^{n / b-(n / p)-s}\right)^{-1} & \text { if } p<b\end{cases}
$$

Now since $\left\{2^{k(s-r)} \gamma_{k}\right\}_{k \in \mathbb{N}} \in \ell_{\min (p, q)}$, we conclude the desired conclusion using (2.28) and (2.29).

Subcase $2.2\left(r \leq n / p_{2}\right.$ and $p>b\left(\right.$ or $s \leq n / p_{2}<r$ and $\left.\left.p>b\right)\right)$. Let $u>0$ satisfy

$$
\max \left(0, \frac{1}{p}-\frac{1}{p_{2}}\right)<\frac{1}{u}<\frac{1}{p_{1}}-\frac{s}{n}
$$


We employ the notations $v, \sigma$, and $\beta$ from (2.13). We have

$$
\left\|\Pi _ { k , 2 } ( f , g ) \left|L_{v}\left\|\leq c|| g\left|B_{p_{2}, \infty}^{r}\|\| f\right| B_{u, \infty}^{\beta}\right\|\left(2^{-k(\beta+r)}\right) .\right.\right.
$$

Since $\left\{2^{-k(\beta+r-\sigma)}\right\}_{k \in \mathbb{N}} \in \ell_{p}$, we can finish the proof of this case using (2.14).

Subcase $2.3\left(n / p_{2}<s<r\right)$. We have only case $p<b$ needs to be verified. As in (2.32), we immediately obtain the result.

Case $3\left(s \geq n / p_{1}\right)$. We have the following subcases.

Subcase $3.1\left(p<p_{2}\right)$. We set $1 / v=1 / p-1 / p_{2}$. Observe that

$$
\begin{aligned}
2^{k s}\left\|\Pi_{k, 2}(f, g) \mid L_{p}\right\| & \leq c 2^{k r} \| \widetilde{\Delta}_{k} g\left|L_{p_{2}}\right| \mid\left(2^{k(s-r)} \sum_{j=0}^{k+1} 2^{j\left(n / p_{1}-n / v\right)}\left\|\Delta_{j} f \mid L_{p_{1}}\right\|\right) \\
& \leq c|g| B_{p_{2}, \infty}^{r}\|\| f \mid B_{p_{1}, \infty}^{n / p_{1}} \|\left(2^{k(s-r)} \sum_{j=0}^{k+1} 2^{-j n / v}\right) .
\end{aligned}
$$

Then, we calculate $\ell_{\min (p, q)}$-norm and conclude the desired estimate by the fact that

$$
\left\{2^{k(s-r)} \sum_{j=0}^{k+1} 2^{-j n / v}\right\}_{k \in \mathbb{N}} \in \ell_{\min (p, q) .}
$$

Subcase $3.2\left(s>n / p_{1}\right.$ and $\left.p \geq p_{2}\right)$. It suffices to apply both embedding $B_{p_{1}, \infty}^{s} \hookrightarrow B_{p_{1}, 1}^{n / p_{1}}$ and (2.29) to

$$
\begin{aligned}
\left\|\Pi_{k, 2}(f, g) \mid L_{p}\right\| & \leq c|| \widetilde{\Delta}_{k} g\left|L_{p}\|\| Q_{k+1} f\right| L_{\infty} \| \\
& \leq c 2^{k\left(n / p_{2}-r-n / p\right)}\left\|g\left|B_{p_{2}, \infty}^{r}\|\| f\right| B_{p_{1}, 1}^{n / p_{1}}\right\| .
\end{aligned}
$$

Subcase $3.3\left(s=n / p_{1}\right.$ and $\left.p \geq p_{2}\right)$. We choose $\alpha>0$ such that $\varepsilon=\alpha-n / p+n / p_{1}+n / p_{2}-$ $r<0$, then it suffices to apply (2.29) to

$$
2^{k n / p_{1}}\left\|\Pi_{k, 2}(f, g)\left|L_{p}\left\|\leq c 2^{k \varepsilon}\right\| g\right| B_{p_{2}, \infty}^{r}\right\|\left\|f \mid B_{p_{1}, \infty}^{n / p_{1}-\alpha}\right\| .
$$

(3) Estimate of $\left\{\Pi_{k, 3}(f, g)\right\}_{k \in \mathbb{N}}$. The proof of this case is obtained similarly to the proof of Theorem 2.1 just by replacing (2.17) and (2.20) with

$$
\begin{gathered}
\max \left(0, \frac{1}{p}-\frac{1}{p_{2}}, \frac{1}{p_{1}}-\frac{r+s}{n}\right)<\frac{1}{u}<\frac{1}{p_{1}}, \\
\max \left(0,1-\frac{1}{p_{2}}, \frac{1}{p}-\frac{1}{p_{2}}\right)<\frac{1}{u}<\frac{1}{p_{1}},
\end{gathered}
$$

respectively.

\section{Multiplication of types $F \cdot B$ and $B \cdot B$}

The next theorem presents a continuation of [3], [5, Theorem 6.1], [6], and [7, Section 5]. 
Theorem 3.1. Let $0<p_{1}<\infty$ (resp., $\left.0<p_{1} \leq \infty\right), 1 \leq p_{2} \leq \infty, 0<q \leq \infty$, and $n / p_{1}-n<$ $s<\min \left(n / p_{1}, n / p_{2}\right)$. Then it holds

$$
F_{p_{1}, q}^{s} \cdot\left(B_{p_{2}, \infty}^{n / p_{2}} \cap L_{\infty}\right) \hookrightarrow F_{p_{1}, q}^{s} \quad\left(\text { resp., } B_{p_{1}, q}^{s} \cdot\left(B_{p_{2}, \infty}^{n / p_{2}} \cap L_{\infty}\right) \hookrightarrow B_{p_{1}, q}^{s}\right) .
$$

Remark 3.2. We note that (3.1), in the F-case, was proved by Franke [4, Section 3.4, Corollary 1] but only in the particular case

$$
p_{2}= \begin{cases}p_{1} & \text { if } 0<p_{1}<2 \\ p_{1}\left(p_{1}-1\right)^{-1} & \text { if } 2 \leq p_{1}<\infty\end{cases}
$$

Also this case yields

$$
F_{p_{1}, q}^{s} \cdot\left(F_{p_{2}, \infty}^{n / p_{2}} \cap L_{\infty}\right) \hookrightarrow F_{p_{1}, q}^{s}
$$

Remark 3.3. Theorem 3.1, when $1 \leq p_{1} \leq p_{2}<\infty$, was proved in [3].

Proof of Theorem 3.1. The estimates of $\left\{\Pi_{k, 1}(f, g)\right\}_{k \in \mathbb{N}}$ and $\left\{\Pi_{k, 3}(f, g)\right\}_{k \in \mathbb{N}}$ are similar to Theorem 2.1, see also [3]. For $\left\{\Pi_{k, 2}(f, g)\right\}_{k \in \mathbb{N}}$ we take, in (2.16), $r=n / p_{2}$, and $q_{2}=\tilde{q}_{2}=$ $\infty$, we obtain (3.1) in the $F$-case. In the $B$-case, we will employ the notations $u, v, \sigma$, and $\beta$ from (2.12) and (2.13) with the modifications $r=n / p_{2}$ and $\sigma=s-n / p_{1}+n / \nu$. One has

$$
2^{k \sigma}\left\|\Pi_{k, 2}(f, g)\left|L_{v}\|\leq c\| g\right| B_{p_{2}, \infty}^{n / p_{2}}\right\|\left(2^{k \beta} \sum_{j=0}^{k+1} 2^{-j \beta} \cdot 2^{j \beta}\left\|\Delta_{j} f \mid L_{u}\right\|\right) .
$$

Since $\beta<0$, the last inequality, in the $\ell_{q}$-norm, is bounded by the expression $c\left\|g \mid B_{p_{2}, \infty}^{n / p_{2}}\right\|$ $\left\|f \mid B_{u, q}^{\beta}\right\|$. At the end, it suffices to use

$$
\ell_{q}^{\sigma}\left(L_{v}^{\gamma}\right) \hookrightarrow \ell_{q}^{s}\left(L_{p_{1}}^{\gamma}\right), \quad B_{p_{1}, q}^{s} \hookrightarrow B_{u, q}^{\beta} .
$$

\section{Some limit cases}

We will prove results of independent interest concerning the limit case for the parameters $s+r$, see [5, Theorems 6.5 and 6.11].

Theorem 4.1. Let $0<p, q, p_{i}, q_{i} \leq \infty,(i=1,2),-\infty<s<\infty$, and $r>0$ such that

$$
\frac{1}{q_{1}}+\frac{1}{q_{2}} \geq 1, \quad s+r=\frac{n}{p_{1}}+\frac{n}{p_{2}}-n>0 .
$$


12 Multiplication in Besov and Lizorkin spaces

If either of the following assertions is satisfied:

(i)

$$
\begin{gathered}
r<\frac{n}{p_{2}}, \quad \frac{1}{p}=\frac{1}{p_{1}}+\frac{1}{p_{2}}-\frac{r}{n}, \quad s<\min \left(\frac{n}{p_{1}}, r\right), \\
\frac{1}{q_{2}} \geq \frac{1}{p_{2}}-\frac{r}{n}, \quad q=\infty, p_{2} \neq \infty,
\end{gathered}
$$

(ii)

$$
\max \left(\frac{1}{p_{1}}, \frac{s}{n}\right)+\max \left(0, \frac{1}{p_{2}}-\frac{r}{n}\right)<\frac{1}{p} \leq \frac{1}{p_{1}}+\frac{1}{p_{2}}
$$

and either of the following cases is satisfied:

(1) $s<r, q_{1} \leq q$,

(2) $s=r, \max \left(q_{1}, q_{2}\right) \leq q$,

then it holds

$$
B_{p_{1}, q_{1}}^{s} \cdot B_{p_{2}, q_{2}}^{r} \hookrightarrow B_{p, q}^{s} \text {. }
$$

Remark 4.2. In Theorem 4.1(i), when $r=n / p_{2}$, we have

$$
B_{p_{1}, q_{1}}^{s} \cdot\left(B_{p_{2}, q_{2}}^{n / p_{2}} \cap L_{\infty}\right) \hookrightarrow B_{p_{1}, q}^{s}
$$

Theorem 4.3. Let $0<p, p_{1}, p_{2}<\infty, 0<q \leq \infty,-\infty<s<\infty$, and $r>0$ such that

$$
s+r=\frac{n}{p_{1}}+\frac{n}{p_{2}}-n>0 .
$$

If either of the following assertions is satisfied:

(i)

$$
r<\frac{n}{p_{2}}, \quad \frac{1}{p}=\frac{1}{p_{1}}+\frac{1}{p_{2}}-\frac{r}{n}, \quad s<\min \left(\frac{n}{p_{1}}, r\right),
$$

(ii)

$$
\max \left(\frac{1}{p_{1}}, \frac{s}{n}\right)+\max \left(0, \frac{1}{p_{2}}-\frac{r}{n}\right)<\frac{1}{p} \leq \frac{1}{p_{1}}+\frac{1}{p_{2}}, \quad s \leq r,
$$

then it holds

$$
F_{p_{1}, q}^{s} \cdot F_{p_{2}, \infty}^{r} \hookrightarrow B_{p, \infty}^{s} .
$$

Remark 4.4. In Theorem 4.3(i), when $r=n / p_{2}$, we have

$$
F_{p_{1}, q}^{s} \cdot\left(F_{p_{2}, \infty}^{n / p_{2}} \cap L_{\infty}\right) \hookrightarrow B_{p_{1}, \infty}^{s} .
$$


Theorem 4.5. Let $0<p, p_{1}<\infty, 0<p_{2}, q_{1}, q_{2} \leq \infty,-\infty<s<\infty$, and $r>0$ such that

$$
q_{1} \geq p_{1}, \quad \frac{1}{q_{1}}+\frac{1}{q_{2}} \geq 1, \quad s<r, \quad s+r=\max \left(0, \frac{n}{p_{1}}+\frac{n}{p_{2}}-n\right) .
$$

If either of the following assertions is satisfied:

(i)

$$
\frac{1}{p}=\frac{1}{p_{1}}+\frac{1}{p_{2}}
$$

(ii)

$$
\max \left(\frac{1}{p_{1}}, \frac{s}{n}\right)+\max \left(0, \frac{1}{p_{2}}-\frac{r}{n}\right)<\frac{1}{p}<\frac{1}{p_{1}}+\frac{1}{p_{2}}
$$

then it holds

$$
F_{p_{1}, q_{1}}^{s} \cdot B_{p_{2}, q_{2}}^{r} \hookrightarrow F_{p, q_{1}}^{s} \text {. }
$$

Proof of Theorem 4.1(i). (i) Estimate of $\left\{\Pi_{k, 1}(f, g)\right\}_{k \in \mathbb{N}}$. We set $1 / u=1 / p_{2}-r / n$. The Hölder inequality and Lemma 2.4 give

$$
2^{k s}|| \Pi_{k, 1}(f, g)\left|L_{p}\|\leq c\| g\right| F_{u, 2}^{0} \|\left(2^{k s}|| \tilde{\Delta}_{k} f\left|L_{p_{1}}\right| \mid\right) .
$$

The embedding $B_{p_{2}, q_{2}}^{r} \hookrightarrow F_{u, 2}^{0}$ together with the $\ell_{\infty}$-norm of (4.15) and $\ell_{q_{1}} \hookrightarrow \ell_{\infty}$ give the desired estimate.

(ii) Estimate of $\left\{\Pi_{k, 2}(f, g)\right\}_{k \in \mathbb{N}}$. Using the notations $u, v, \sigma$, and $\beta$ from (2.12) and (2.13), we have, as in (2.15),

$$
2^{k \sigma}\left\|\left\{\Pi_{k, 2}(f, g)\right\}_{k \in \mathbb{N}}\left|\ell_{\infty}\left(L_{v}\right)\|\leq c\| g\right| B_{p_{2}, \infty}^{r}\right\|\left\|f \mid B_{u, \infty}^{\beta}\right\|
$$

and the conclusion is obtained by (3.5).

(iii) Estimate of $\left\{\Pi_{k, 3}(f, g)\right\}_{k \in \mathbb{N}}$. We set $1 / b=1 / p_{1}+1 / p_{2}$. By Lemma 1.6 and the Hölder inequality, we obtain

$$
\left\|\Pi_{k, 3}(f, g) \mid L_{b}\right\| \leq c 2^{-k(s+r)} \sum_{j=k}^{\infty}\left(2^{j r} \| \bar{\Delta}_{j} g\left|L_{p_{2}}\right| \mid\right)\left(2^{j s}\left\|\Delta_{j} f \mid L_{p_{1}}\right\|\right) .
$$

Using $\ell_{d} \hookrightarrow \ell_{1}$ (with $1 / d=1 / q_{1}+1 / q_{2}$ ) we employ the Hölder inequality again to conclude that the last term of (4.17) is bounded by $c\left\|g\left|B_{p_{2}, q_{2}}^{r}\|\| f\right| B_{p_{1}, q_{1}}^{s}\right\|$. We finish the proof of this case by applying the embedding $\ell_{\infty}^{s+r}\left(L_{b}^{\gamma}\right) \hookrightarrow \ell_{\infty}^{s}\left(L_{p}^{\gamma}\right)$.

Proof of Theorem 4.1(ii). For $\left\{\Pi_{k, 1}(f, g)\right\}_{k \in \mathbb{N}}$ and $\left\{\Pi_{k, 2}(f, g)\right\}_{k \in \mathbb{N}}$, we can use the same methods in Theorem 2.5, see also [10, Sections 4.4.3 and 4.4.4]. 
Estimate of $\left\{\Pi_{k, 3}(f, g)\right\}_{k \in \mathbb{N}}$. By Lemmas 1.6, 1.8 and the Hölder inequality, we have

$$
2^{k s}|| \Pi_{k, 3}(f, g) \mid L_{p} \| \leq c 2^{k\left(n / p_{1}+n / p_{2}-n / p-r\right)} \sum_{j=k}^{\infty}\left(2^{j r}|| \bar{\Delta}_{j} g\left|L_{p_{2}}\right| \mid\right)\left(2^{j s}|| \Delta_{j} f\left|L_{p_{1}}\right| \mid\right) \text {. }
$$

Since $n / p_{1}+n / p_{2}-n / p-r<0$, we conclude the desired estimate using $\ell_{d} \hookrightarrow \ell_{1}$ (with $\left.1 / d=1 / q_{1}+1 / q_{2}\right)$.

Proof of Theorem 4.3(i). (i) Estimate of $\left\{\Pi_{k, 1}(f, g)\right\}_{k \in \mathbb{N}}$. We set $1 / u=1 / p_{2}-r / n$, (i.e., $\left.1 / p=1 / p_{1}-1 / u\right)$. As in (2.9), the choice of $a_{1}>n / u$ and $a_{2}>n / p_{1}$ leads to

$$
\begin{aligned}
\left\|\left\{2^{k s} \Pi_{k, 1}(f, g)\right\}_{k \in \mathbb{N}} \mid \ell_{\infty}\left(L_{p}\right)\right\| & \leq c\left\|\sup _{j \in \mathbb{N}} Q_{j}^{*, a_{1}} g\left|L_{u}\|\|\left\{2^{k s} \tilde{\Delta}_{k}^{*, a_{2}} f\right\}_{k \in \mathbb{N}}\right| \ell_{\infty}\left(L_{p_{1}}\right)\right\| \\
& \leq c\left\|g\left|F_{u, 2}^{0}\|\| f\right| B_{p_{1}, \infty}^{s}\right\| .
\end{aligned}
$$

We conclude the desired estimate by applying both $F_{p_{2}, \infty}^{r} \hookrightarrow F_{u, 2}^{0}$ and $F_{p_{1}, \infty}^{s} \hookrightarrow B_{p_{1}, \infty}^{s}$.

(ii) Estimate of $\left\{\Pi_{k, 2}(f, g)\right\}_{k \in \mathbb{N}}$. Using the notations $u, v, \sigma$, and $\beta$ from (2.12) and (2.13), we have

$$
2^{k \sigma}\left|\Pi_{k, 2}(f, g)\right| \leq c \sup _{\ell \in \mathbb{N}}\left(2^{\ell r} \Delta_{\ell}^{*, a_{1}} g\right)\left(2^{k \beta} \sum_{j=0}^{k+1} 2^{-j \beta}\left(2^{j \beta} \Delta_{j}^{*, a_{2}} f\right)\right) .
$$

Since $\beta<0$, then

$$
\left\|\left\{2^{k \sigma} \Pi_{k, 2}(f, g)\right\}_{k \in \mathbb{N}}\left|\ell_{\infty}\left\|\leq c \sup _{\ell \in \mathbb{N}}\left(2^{\ell r} \bar{\Delta}_{\ell}^{*, a_{1}} g\right)\right\|\left\{2^{j \beta} \Delta_{j}^{*, a_{2}} f\right\}_{j \in \mathbb{N}}\right| \ell_{\infty}\right\| .
$$

We choose $a_{1}>n / p_{2}$ and $a_{2}>n / u$. We obtain the desired result by applying the Hölder inequality, the embeddings $L_{v}^{\gamma}\left(\ell_{\infty}^{\sigma}\right) \hookrightarrow L_{p}^{\gamma}\left(\ell_{\infty}^{s}\right) \hookrightarrow \ell_{\infty}^{s}\left(L_{p}^{\gamma}\right)$ and $F_{p_{1}, q}^{s} \hookrightarrow F_{u, \infty}^{\beta}$.

(iii) Estimate of $\left\{\Pi_{k, 3}(f, g)\right\}_{k \in \mathbb{N}}$. We set $1 / u=1 / p_{2}+1 / p_{1}$. We begin by the inequality

$$
\left\|\sum_{k=0}^{\infty} \Pi_{k, 3}(f, g)\left|B_{p, \infty}^{s}\|\leq c\| \sum_{j=0}^{\infty} Q_{j}\left(\bar{\Delta}_{j} g \cdot \Delta_{j} f\right)\right| B_{u, \infty}^{s+r}\right\| .
$$

We can write

$$
\left|Q_{j}\left(\bar{\Delta}_{j} g \cdot \Delta_{j} f\right)\right| \leq c \sup _{j \in \mathbb{N}}\left(\Delta_{j}^{*, a_{1}} g \cdot \Delta_{j}^{*, a_{2}} f\right) .
$$

We choose $a_{1}>n / p_{2}$ and $a_{2}>n / \min \left(p_{1}, q\right)$. Then Lemma 1.7 gives the correct bound for (4.22).

The same method works for the proofs of Theorems 4.3(ii) and 4.5. We omit the details. 
Remark 4.6. Theorems 4.1(i) and 4.3(i), when $1 \leq p \leq \infty$, were proved by Johnsen in [5, Theorems 6.11 and 6.5], respectively.

\section{Application}

We consider $S_{1,0}^{0}(E)(E$ a Banach space), the class of symbols $(x, \xi) \rightarrow a(x, \xi)$ satisfying

$$
\| \partial_{\xi}^{\beta} a(\cdot, \xi)|E| \mid \leq c_{\beta}(1+|\xi|)^{-|\beta|} \quad\left(\forall \beta \in \mathbb{N}^{n}\right),
$$

and we define the pseudodifferential operator by the formula

$$
\mathrm{Op}_{a} f(x)=(2 \pi)^{-n} \int_{\mathbb{R}^{n}} e^{i x \cdot \xi} a(x, \xi) \hat{f}(\xi) \mathrm{d} \xi \quad\left(\forall f \in \mathscr{Y}, \forall x \in \mathbb{R}^{n}\right)
$$

As mentioned in the introduction, the theorems of this section present an application of the previous results in this paper.

Theorem 5.1. Let $1 \leq p, p_{1}, p_{2}, q, q_{2} \leq \infty,-\infty<s<\infty$, and $r>0$. Under the hypotheses of Theorem 2.1 (with $p_{2} \neq \infty$ and $r<n / p_{2}$ ) or Theorem 2.5, the operator $\mathrm{Op}_{a}$ is bounded from $F_{p_{1}, q}^{s}$ to $F_{p, q}^{s}$, for all $a \in S_{1,0}^{0}\left(B_{p_{2}, q_{2}}^{r}\right)$.

The proof of Theorem 5.1 is based on the following almost-orthogonality lemma.

Lemma 5.2. Let $\gamma>1$ and let $p, p_{1}, p_{2}, q, q_{2}, r, s$ be the same as in Theorem 2.1 (with $p_{2} \neq \infty$ and $\left.r<n / p_{2}\right)$ or Theorem 2.5. For all sequences $\left\{m_{j}\right\}_{j \in \mathbb{N}} \subset B_{p_{2}, q_{2}}^{r}$ and all sequences $\left\{f_{j}\right\}_{j \in \mathbb{N}}$ of functions such that $\operatorname{supp} \hat{f}_{j} \subset\left\{\xi \in \mathbb{R}^{n}: \gamma^{-1} 2^{j} \leq|\xi| \leq \gamma 2^{j}\right\}$, the estimate

$$
\left\|\sum_{j=0}^{\infty} m_{j} \cdot f_{j}\left|F_{p, q}^{s}\|\leq c\|\left\{f_{j}\right\}_{j \in \mathbb{N}}\right| L_{p_{1}}^{\gamma}\left(\ell_{q}^{s}\right)\right\|
$$

holds with $c=c^{\prime} \sup _{j \geq 0}\left\|m_{j} \mid B_{p_{2}, q_{2}}^{r}\right\|$.

Proof. Observe that $\Delta_{k} f_{j} \neq 0$ and $Q_{k+1} f_{j} \neq 0$ if $k-N \leq j \leq k+N+2$ and $j \leq k+N+2$, respectively, where $N=\left[\log _{2} \gamma\right]$; (here $[x]$ denotes the greatest integer less than or equal to $x$ ). Then it suffices to apply Theorem 2.1 (and/or Theorem 2.5) to the following decomposition:

$$
\Delta_{k}\left(\sum_{j=0}^{\infty} m_{j} \cdot f_{j}\right)=\sum_{\ell=-N}^{N+2} \Pi_{k, 1}\left(m_{k+\ell}, f_{k+\ell}\right)+\sum_{j=0}^{k+N+2} \Pi_{k, 2}\left(m_{j}, f_{j}\right)+\sum_{\ell=-N}^{N+2} \tilde{\Pi}_{k, 3}\left(m_{\cdot+\ell}, f_{\cdot+\ell}\right),
$$

where $\tilde{\Pi}_{k, 3}\left(m_{\cdot+\ell}, f_{\cdot+\ell}\right)=\sum_{j=k}^{\infty} \Delta_{k}\left(\bar{\Delta}_{j} m_{j+\ell} \cdot \Delta_{j} f_{j+\ell}\right)$ (see also (2.7)).

Proof of Theorem 5.1. We begin by writing

$$
a(x, \xi)=(2 \pi)^{-n} \int_{\mathbb{R}^{n}}\left(1+|u|^{2}\right)^{-(n+1) / 2} a_{u}(x, \xi) d u+\lambda(x, \xi)
$$


where $\lambda(x, \xi)=0$ for $|\xi| \geq 3$,

$$
\begin{gathered}
\left\|\partial_{\xi}^{\beta} \lambda(\cdot, \xi) \mid B_{p_{2}, q_{2}}^{r}\right\| \leq c_{\beta}(1+|\xi|)^{-|\beta|} \quad\left(\forall \beta \in \mathbb{N}^{n}\right), \\
a_{u}(x, \xi)=\sum_{j=0}^{\infty} m_{j, u}(x) \theta_{u}\left(2^{-j} \xi\right), \\
\sup _{j \in \mathbb{N}, u \in \mathbb{R}^{n}}\left\|m_{j, u} \mid B_{p_{2}, q_{2}}^{r}\right\| \leq c, \\
\theta_{u}(\xi)=(2 \pi)^{-n}\left(1+|u|^{2}\right)^{(n+1-L) / 2} e^{i u \cdot \xi} \theta(\xi),
\end{gathered}
$$

$\theta$ is a $C^{\infty}$ function with $\operatorname{supp} \theta \subset\left\{\xi \in \mathbb{R}^{n}: 1 \leq|\xi| \leq 3\right\}$, and

$$
\left\|\theta_{u}^{(\beta)} \mid L_{\infty}\right\| \leq c \quad\left(\forall u \in \mathbb{R}^{n},|\beta| \leq L-n-1\right) .
$$

For the decomposition (5.5), we refer the reader to [2] or [8].

Now, by Lemma 5.2, we have

$$
\left\|\mathrm{Op}_{a_{u}} f\left|F_{p, q}^{s}\|\leq c\|\left\{2^{j s} f_{u, j}\right\}_{j \in \mathbb{N}}\right| L_{p_{1}}\left(\ell_{q}\right)\right\| \leq c^{\prime}\left\|f \mid F_{p_{1}, q}^{s}\right\|
$$

where $\mathscr{F}\left(f_{u, j}\right)(\xi)=\theta_{u}\left(2^{-j} \xi\right) \hat{f}(\xi)$ and $c^{\prime}$ is independent of $u$. Next, we can write

$$
\mathrm{Op}_{\lambda} f(x)=\int_{\mathbb{R}^{n}}\left(1+|u|^{2}\right)^{-(n+1) / 2} b_{u}(x) f(x+u) d u,
$$

where

$$
b_{u}(x)=(2 \pi)^{-n} \int_{\mathbb{R}^{n}} e^{-i u \cdot \xi}(I-\Delta)^{2 n} \lambda(x, \xi) d \xi
$$

Theorems 2.1 and 2.5 immediately give

$$
\begin{aligned}
\left\|\mathrm{Op}_{\lambda} f \mid F_{p, q}^{s}\right\| & \leq \sup _{u \in \mathbb{R}^{n}}\left\|b_{u} \cdot f(\cdot+u) \mid F_{p, q}^{s}\right\| \\
& \leq\left(\sup _{u \in \mathbb{R}^{n}}\left\|b_{u} \mid B_{p_{2}, q_{2}}^{r}\right\|\right)\left\|f\left|F_{p_{1}, q}^{s}\|\leq c\| f\right| F_{p_{1}, q}^{s}\right\| .
\end{aligned}
$$

Theorem 5.3. Let $1 \leq p, p_{1}, q, q_{1} \leq \infty, r>0$, and

$$
-r+\frac{n}{p}+\frac{n}{p_{1}}-n<s<\min \left(\frac{n}{p}, r\right)
$$

Suppose that $a \in S_{1,0}^{0}\left(B_{p_{1}, q_{1}}^{r}\right)$ if $r>n / p_{1}$ and $a \in S_{1,0}^{0}\left(L_{\infty}\right) \cap S_{1,0}^{0}\left(B_{p_{1}, \infty}^{n / p_{1}}\right)$ if $r=n / p_{1}$. Then the operator $\mathrm{Op}_{a}$ is bounded on $F_{p, q}^{s}$ and $B_{p, q}^{s}$. 
For the proof, we apply Theorem 3.1 and proceed as in Theorem 5.1, however, we need an almost-orthogonality estimate of the type in Lemma 5.2, that is, the following lemma.

Lemma 5.4. Let $\gamma>1,0<p, p_{1}, q, q_{1} \leq \infty, r \geq n / p_{1}$, and $s$ be as in Theorem 5.3. For all sequences of functions $\left\{f_{j}\right\}_{j \in \mathbb{N}}$ such that supp $\hat{f}_{j} \subset\left\{\xi \in \mathbb{R}^{n}: \gamma^{-1} 2^{j} \leq|\xi| \leq \gamma 2^{j}\right\}$ and all sequences $\left\{m_{j}\right\}_{j \in \mathbb{N}} \subset B_{p_{1}, q_{1}}^{r}$ (or $\left.\left\{m_{j}\right\}_{j \in \mathbb{N}} \subset B_{p_{1}, q_{1}}^{n / p_{1}} \cap L_{\infty}\right)$, the estimates

$$
\begin{aligned}
& \left\|\sum_{j=0}^{\infty} m_{j} \cdot f_{j}\left|F_{p, q}^{s}\|\leq c\|\left\{f_{j}\right\}_{j \in \mathbb{N}}\right| L_{p}^{\gamma}\left(\ell_{q}^{s}\right)\right\|, \\
& \left\|\sum_{j=0}^{\infty} m_{j} \cdot f_{j}\left|B_{p, q}^{s}\left\|\leq c^{\prime}\right\|\left\{f_{j}\right\}_{j \in \mathbb{N}}\right| \ell_{q}^{s}\left(L_{p}^{\gamma}\right)\right\|,
\end{aligned}
$$

hold. The constants $c$ and $c^{\prime}$ are of the form $c^{\prime \prime} \sup _{j \in \mathbb{N}}\left(\left\|m_{j}\left|L_{\infty}\|+\| m_{j}\right| B_{p_{1}, q_{1}}^{r}\right\|\right)$.

\section{Acknowledgment}

We would like to thank the referee(s) for the most helpful remarks and corrections which led to the improvements, the results, and the presentation of this paper.

\section{References}

[1] J. Bergh and J. Löfström, Interpolation Spaces. An Introduction, Springer, Berlin, 1976.

[2] G. Bourdaud and M. Moussai, Continuité des commutateurs d'intégrales singulières sur les espaces de Besov, Bulletin des Sciences Mathématiques 118 (1994), no. 2, 117-130.

[3] D. Drihem and M. Moussai, Some embeddings into the multiplier spaces associated to Besov and Lizorkin-Triebel spaces, Zeitschrift für Analysis und ihre Anwendungen 21 (2002), no. 1, 179184.

[4] J. Franke, On the spaces $\mathbf{F}_{p q}^{s}$ of Triebel-Lizorkin type: pointwise multipliers and spaces on domains, Mathematische Nachrichten 125 (1986), 29-68.

[5] J. Johnsen, Pointwise multiplication of Besov and Triebel-Lizorkin spaces, Mathematische Nachrichten 175 (1995), 85-133.

[6] J. Marschall, On the boundedness and compactness of nonregular pseudo-differential operators, Mathematische Nachrichten 175 (1995), 231-262.

[7] _ Nonregular pseudo-differential operators, Zeitschrift für Analysis und ihre Anwendungen 15 (1996), no. 1, 109-148.

[8] M. Moussai, Continuity of pseudo-differential operators on Bessel and Besov spaces, Serdica. Mathematical Journal 27 (2001), no. 3, 249-262.

[9] J. Peetre, New Thoughts on Besov Spaces, Duke University Mathematics Series, no. 1, Mathematics Department, Duke University, North Carolina, 1976.

[10] T. Runst and W. Sickel, Sobolev Spaces of Fractional Order, Nemytskij Operators, and Nonlinear Partial Differential Equations, de Gruyter Series in Nonlinear Analysis and Applications, vol. 3, Walter de Gruyter, Berlin, 1996.

[11] H. Triebel, Interpolation Theory, Function Spaces, Differential Operators, North-Holland Mathematical Library, vol. 18, North-Holland, Amsterdam, 1978.

[12] _ Theory of Function Spaces, Monographs in Mathematics, vol. 78, Birkhäuser, Basel, 1983. 


\section{Multiplication in Besov and Lizorkin spaces}

[13] _ Theory of Function Spaces. II, Monographs in Mathematics, vol. 84, Birkhäuser, Basel, 1992.

[14] M. Yamazaki, A quasi-homogeneous version of paradifferential operators. I. Boundedness on spaces of Besov type, Journal of the Faculty of Science. University of Tokyo. Section IA. Mathematics 33 (1986), no. 1, 131-174.

Douadi Drihem: Department of Mathematics, Laboratory of Mathematics Pure and Applied, M'Sila University, P.O. Box 166, M'Sila 28000, Algeria

E-mail address: douadidr@yahoo.fr

Madani Moussai: Department of Mathematics, Laboratory of Mathematics Pure and Applied, M'Sila University, P.O. Box 166, M'Sila 28000, Algeria

E-mail address: mmoussai@yahoo.fr 


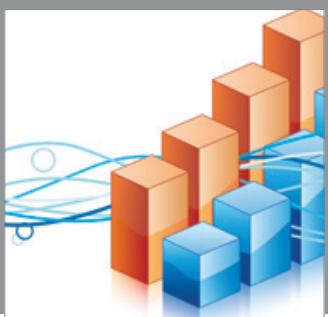

Advances in

Operations Research

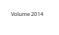

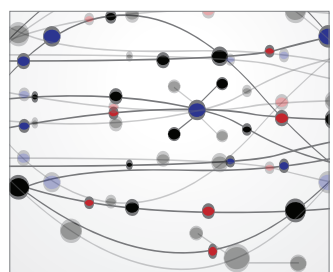

\section{The Scientific} World Journal
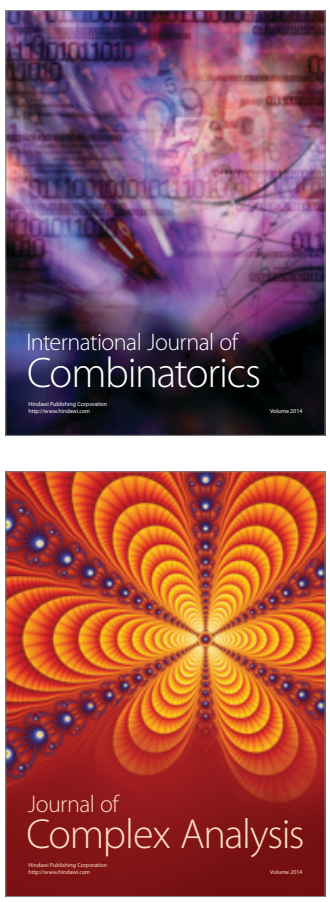

International Journal of

Mathematics and

Mathematical

Sciences
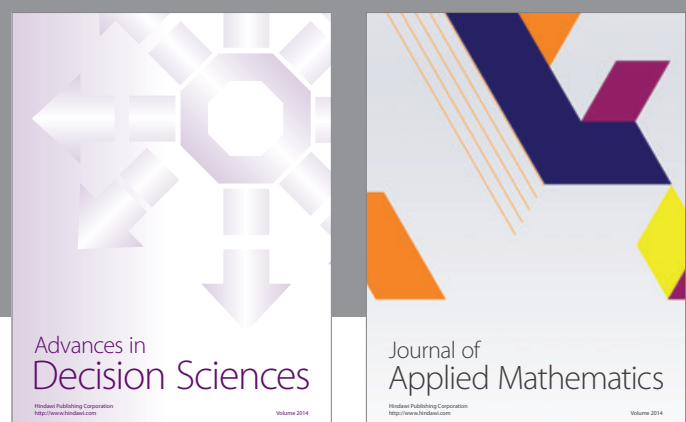

Journal of

Applied Mathematics
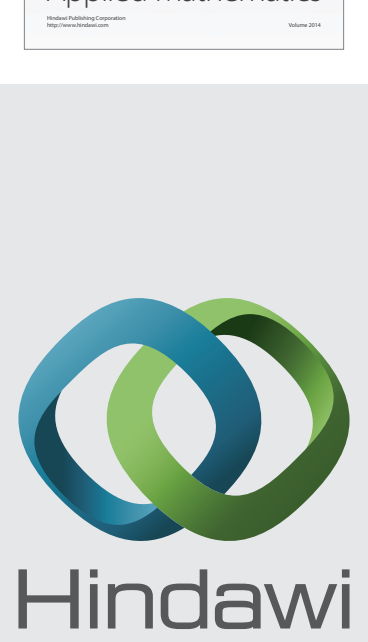

Submit your manuscripts at http://www.hindawi.com
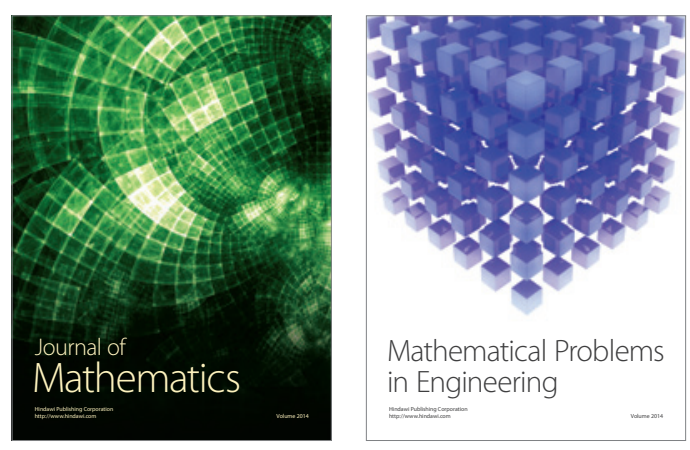

Mathematical Problems in Engineering
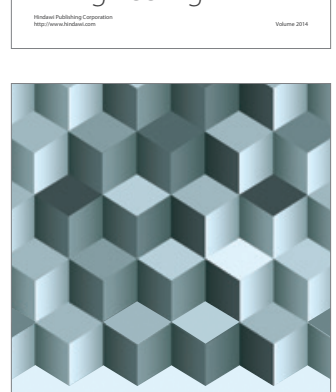

Journal of

Function Spaces
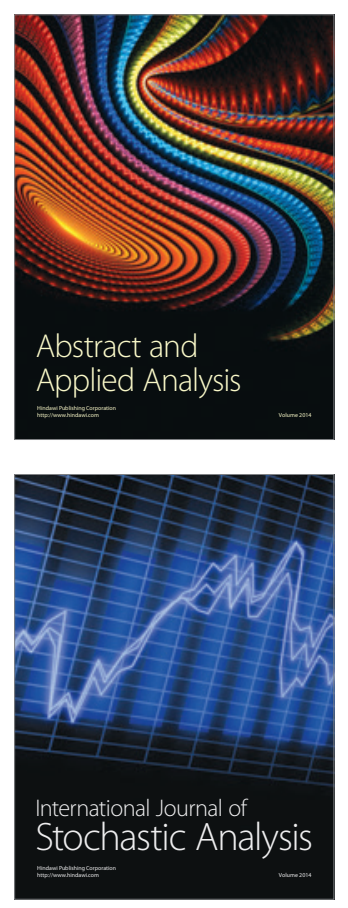

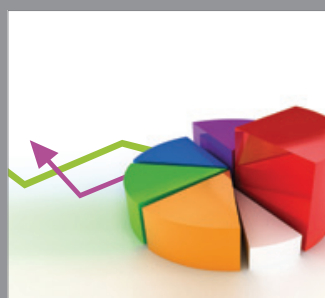

ournal of

Probability and Statistics

Promensencen
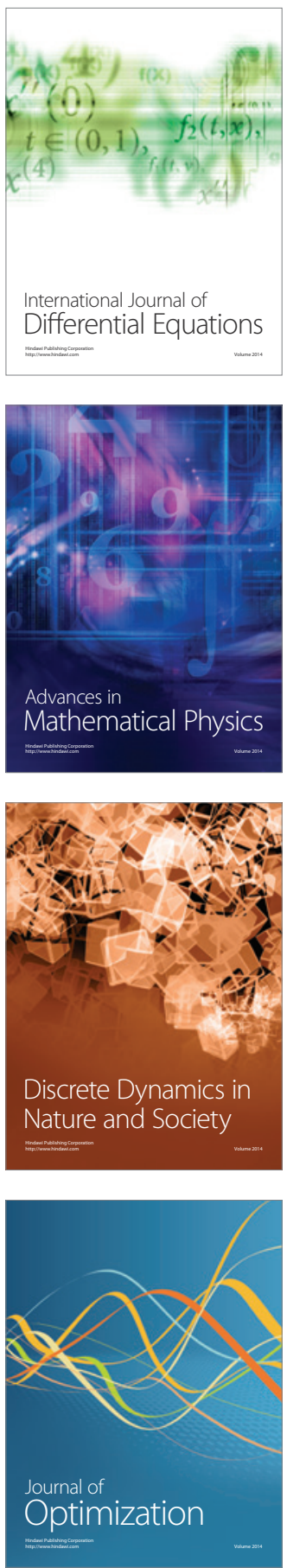